\title{
Organellar stress intersects the astrocyte endoplasmic reticulum, mitochondria and nucleolus in HIV associated neurodegeneration
}

\author{
Shruthi Nooka ${ }^{1}$ and Anuja Ghorpade ${ }^{1}$
}

Human immunodeficiency virus (HIV)-1 infection is no longer a death sentence given the success of antiretroviral (ARV) therapy (ART). HIV-1 invades the central nervous system (CNS) early in infection and over the long run causes neurodegeneration. Despite the dramatic reductions in the severity of these neurocognitive effects in the post-ART era, mild to moderate motor and cognitive impairments persist in $\mathrm{HIV}$-infected individuals that are collectively termed HIV-associated neurocognitive disorders (HAND). While we await the HIV cure, effective adjunctive therapies are needed to target indirect pathological mechanisms of HIV infection that persists in the CNS, especially for cells that are silent for virus production and thus resistant to ART. HIV hijacks the host intracellular and intercellular communication highways, exacerbating neuropathogenesis. Persistent low-level inflammation and oxidative stress contribute to pathological damage in many neurological disorders, including those associated with HIV. One of the principal CNS cell types mediating these events are astrocytes. Thus, further investigations uncovering novel HIV-induced neurotoxic mechanisms in astrocytes and their contributions to HAND are timely.

Eukaryotic cells contain several intracellular membranebound organelles with specialized functions. An ongoing dialog between the endoplasmic reticulum (ER), mitochondria, nucleus, Golgi apparatus and others is pivotal for cellular function in both homeostasis and disease. Given that ultimately all human diseases have a cellular and/or molecular basis, cellular stress responses and

Correspondence: Anuja Ghorpade (Anuja.Ghorpade@unthsc.edu)

'Department of Microbiology, Immunology and Genetics, University of North Texas Health Science Center, Fort Worth, TX, USA dialog play key mechanistic roles. Emerging evidence emphasizes organelle communication linking to human pathologies ${ }^{1}$. The challenge is to decipher the multifaceted signaling network of crosstalk among these cellular compartments leading to malfunction and possibly death, which are necessary for identifying specific therapeutic approaches for neurodegenerative diseases.

The mechanisms underlying neurodegenerative disorders are multifactorial and include cellular insults such as oxidative stress, neuroinflammation, mitochondrial dysfunction, excitotoxicity, and accumulation of misfolded proteins ${ }^{2}$. To cope with such stress, cells have established an extensive range of complex response mechanisms that act at the organelle level. In the ER, the unfolded protein response (UPR) is generally initiated by physiological and pathological insults such as high protein demand, viral infections, inflammation, and overload of mutant proteins. Over the last decade, UPR has been implicated in many neurodegenerative diseases ${ }^{3}$. More recently, UPR served as a therapeutic target for treatment and prevention of neurodegeneration by inhibiting the function of ER stress-specific mediators ${ }^{4}$. However, the effect of HIV infection on UPR in general, and particularly in astrocytes, has scarcely been investigated. A significant increase in the ER chaperone BiP, binding immunoglobulin protein expression was upregulated in the gray matter of mid-frontal cortex of patients with HIV infection $^{5}$. Our data revealed that HIV-associated inflammation significantly increased expression of the ER stress markers $\mathrm{BiP}$ and CCAAT-enhancer-binding protein homologous protein $(\mathrm{CHOP})^{6}$. We also showed activation of all three UPR pathways in human astrocytes in response to inflammation, thus highlighting mutual 
regulation between ER-mediated UPR and inflammation during HIV-1 CNS infection ${ }^{6}$.

The neurotoxic side effects of ARV drugs are among several contributing factors to the continued prevalence of $\mathrm{HAND}^{7}$. However, ARV drug-mediated toxicity in CNS, particularly in glial cells, remains largely unexplored. Our current data provide initial evidence that ARV drugs, specifically nucleoside reverse transcriptase inhibitors (NRTIs), such as abacavir, trigger astrocyte ER stress responses ${ }^{6}$. This knowledge will help guide the development of treatment strategies aimed at reducing antiretroviral neurotoxicity.

Recent findings revealed that the junction between the ER and the mitochondria plays a critical role in cell death regulation ${ }^{8}$. Increased oxidative stress and calcium dysregulation involving the mitochondria and ER are pivotal in neuropathogenesis ${ }^{9}$. HIV proteins gp120 and Tat disrupt calcium-regulating systems in the plasma membrane and ER in neurons. Disturbances in calcium homeostasis induce injury and/or death in neurons and glia that ultimately result in tissue loss in HIV-vulnerable brain regions. Calcium dysregulation is also implicated in longterm neurodegenerative disorders including Alzheimer's, Parkinson's, and Huntington's diseases ${ }^{10}$.

The ER serves as the main storage organelle for calcium. Our studies showed that a prototypical neuroinflammatory cytokine interleukin (IL)- $1 \beta$ and abacavir induced intracellular calcium signaling in astrocytes and upregulated calnexin expression, a calcium-dependent ER chaperone $^{6}$. Chronic elevated cytosolic calcium in astrocytes can be toxic causing neuronal injury or death. Intracellular calcium chelation reduced HIV-associated astrocyte ER stress, indicating a mechanistic role for calcium dysregulation ${ }^{6}$. Since excessively increased astrocyte cytosolic calcium can be toxic and cause neuronal injury and death, our data suggest that both abacavir and neuroinflammation potentially play a role in development of HAND during long-term ART treatment in HIV+ individuals. Mitochondrial dysfunction is also linked to both HIV and ART. Among ARV drugs, NRTIs are known to induce mitochondrial dysfunction by interfering with mitochondrial DNA replication. Since the ER and mitochondria interact closely with each other, sustained ER stress and calcium release can induce membrane permeability transition pore (mPTP) opening on mitochondria $^{11}$. Mitochondrial dysfunction plays a significant role in several neurodegenerative diseases ${ }^{12}$. In our studies, intracellular calcium chelation significantly reduced IL-1 $\beta$ and ARV-dependent mPTP opening: clearly linking calcium-mediated ER stress to mitochondrial dysfunction ${ }^{6}$.

Nucleolar stress, a novel and relatively less studied component of neurodegenerative processes, is associated with impaired rRNA transcription ${ }^{13}$. HIV-1-mediated nucleolar stress is not well documented. Cells combat ER stress, regardless of its source, by inhibiting ribosome synthesis and attenuating protein translation. Since oxidative stress is also implicated in neurodegenerative diseases ${ }^{14}$, we assayed regulator of ribosome synthesis 1 (RRS1) levels in response to inflammation and hydrogen peroxide $\left(\mathrm{H}_{2} \mathrm{O}_{2}\right)$ (Fig. 1a-c). As a protein that inhibits transcription of both rRNA and ribosomal protein genes, elevated expression of RRS1 indicates that HIV-relevant stimuli augment nucleolar stress in astrocytes.

Astrocyte elevated gene (AEG)-1 was identified as a novel modulator of astrocyte responses to injury and HIV-associated neuroinflammation, signifying central regulatory role for AEG-1 in HAND. In addition, AEG-1 translocated to nucleolus in response to injury and oxidative stress ${ }^{15}$. In the context of our previously published works, we postulated that AEG-1 likely contributes to HIV-1/ARV-induced ER stress in astrocytes. We confirmed AEG-1 as an ER stress inducible gene ${ }^{6}$. To the best of our knowledge, we are the first to report interaction between AEG-1 and calnexin, a calcium-binding chaperone. This comes as no surprise though, as AEG-1 is a multifunctional scaffolding protein. It is enticing to anticipate that AEG-1 also regulates ER-dependent calcium signaling through calnexin.

Together these data indicate that organellar stress is intertwined between the ER, mitochondria, and nucleolus in astrocytes to mediate HIV-associated neurodegeneration (Fig. 1d). HIV, inflammation, and ART elevate intracellular calcium to activate the UPR and mitochondrial dysfunction in astrocytes. HIV-associated inflammation and oxidative stress also initiate nucleolar stress to mitigate protein overload and ER stress in astrocytes during HIV-1 CNS infection. Our work also revealed that AEG-1 likely occupies a unique niche in HIV-associated organellar stress, signifying its contribution towards neurodegeneration. AEG-1 functional range is connected to localization to various subcellular compartments including the nucleus, nucleolus, and ER, making AEG-1 a wide-ranging therapeutic target that mediates astrocyte responses. Taken together, this study provides a framework for further elucidation of intersecting organellar stress responses in HIV-1 neuropathogenesis in order to develop novel therapeutic targets for HAND. Given that these works focus on astrocytes with a central role in all CNS injuries, they shed unique light on the web of organellar stress pathways and have far-reaching implications to neurodegeneration beyond that associated with HIV.

\section{Acknowledgements}

This study was funded by the National Institute on Mental Health (R01MH087345) to A.G. We appreciate the assistance of the Laboratory of Developmental Biology for providing human brain tissues; supported by NIH 


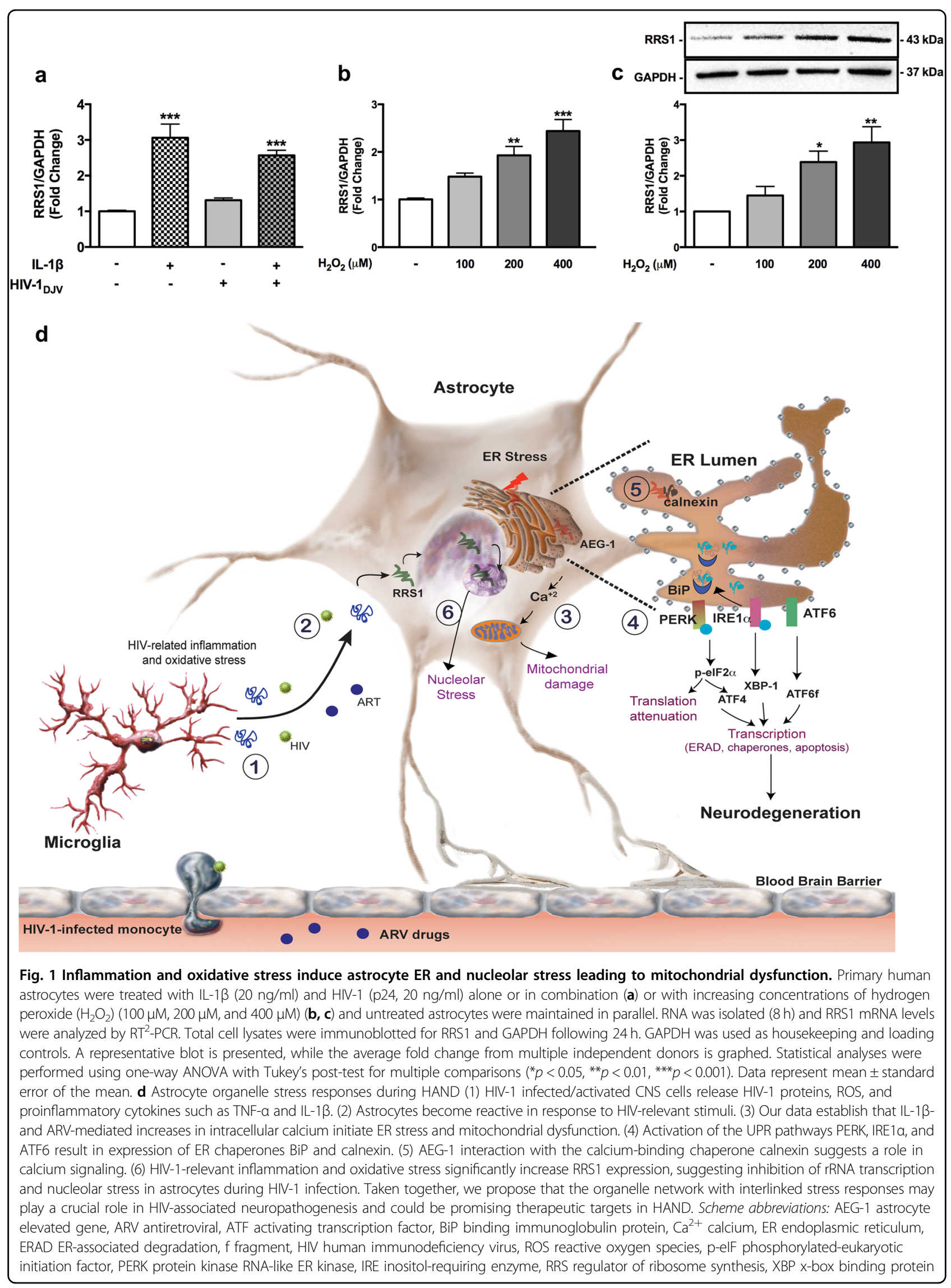


R24 HD0008836 from the Eunice Kennedy Shriver National Institute of Child Health \& Human Development.

\section{Conflict of interest}

The authors declare that they have no conflict of interest.

\section{Publisher's note}

Springer Nature remains neutral with regard to jurisdictional claims in published maps and institutional affiliations.

Received: 31 July 2017 Revised: 10 January 2018 Accepted: 11 January 2018 Published online: 22 February 2018

\section{References}

1. Bravo-Sagua, R. et al. Organelle communication: signaling crossroads between homeostasis and disease. Int J Biochem Cell Biol. 50, 55-59 (2014).

2. Chaudhari, N. et al. A molecular web: endoplasmic reticulum stress, inflammation, and oxidative stress. Front Cell Neurosci. 8, 213 (2014).

3. Scheper $W$, et al. The unfolded protein response in neurodegenerative diseases: a neuropathological perspective. Acta Neuropathol. 130, 315-331 (2015).

4. Hetz, C. et al. Targeting the unfolded protein response in disease. Nat Rev Drug Discov. 12, 703-719 (2013).
5. Lindl, K. A. et al. Expression of the endoplasmic reticulum stress response marker, BiP, in the central nervous system of HIV-positive individuals. Neuropathol Appl Neurobiol. 33, 658-669 (2007).

6. Nooka, S. et al. HIV-1-associated inflammation and antiretroviral therapy regulate astrocyte endoplasmic reticulum stress responses. Cell Death Discov. 3, 17061 (2017).

7. Robertson, K. et al. Antiretroviral neurotoxicity. J Neurovirol. 18, 388-399 (2012).

8. Grimm S. The ER-mitochondria interface: the social network of cell death Biochim Biophys Acta. 1823, 327-334 (2012).

9. Cali, T. et al. Calcium and endoplasmic reticulum-mitochondria tethering in neurodegeneration. DNA Cell Biol. 32,140-146 (2013).

10. Zundorf, G. et al. Calcium dysregulation and homeostasis of neural calcium in the molecular mechanisms of neurodegenerative diseases provide multiple targets for neuroprotection. Antioxid Redox Signal. 14, 1275-1288 (2011).

11. Rasola, A. et al. Mitochondrial permeability transition in $\mathrm{Ca}(2+)$-dependent apoptosis and necrosis. Cell Calcium. 50, 222-233 (2011).

12. Reddy, P. H. et al. Mitochondria as a therapeutic target for aging and neurodegenerative diseases. Curr Alzheimer Res. 8, 393-409 (2011).

13. Parlato, R. et al. Nucleolar activity in neurodegenerative diseases: a missing piece of the puzzle? J Mol. Med. (Berl.). 91, 541-547 (2013).

14. Uttara, B. et al. Oxidative stress and neurodegenerative diseases: a review of upstream and downstream antioxidant therapeutic options. Curr Neuropharmacol. 7, 65-74 (2009).

15. Vartak-Sharma, N. et al. Astrocyte elevated gene-1 (AEG-1) and the A(E)Ging HIV/AIDS-HAND. Prog. Neurobiol. 157, 133-157 (2017). 